\title{
Assessment of Genetic Diversity among Twenty Indian Wheat (Triticum aestivum L.) Cultivars using Simple Sequence Repeat (SSR) Markers
}

\author{
Vandana Sharma ${ }^{1}$, Vaishali $^{1 *}$, Pushpendra Kumar ${ }^{1}$, \\ Manoj Kumar Yadav ${ }^{1}$ and Pooran Chand ${ }^{2}$
}

${ }^{1}$ Department of Agriculture Biotechnology, ${ }^{2}$ Department of Genetics and Plant Breeding, Sardar Vallabhbhai Patel University of Agriculture and Technology, Meerut-250 110 (U.P), India

*Corresponding author

\section{A B S T R A C T}

\section{Keywords}

Bread wheat, Genetic diversity, SSR, PIC,

Resolving power

Article Info

Accepted:

16 February 2018

Available Online:

10 March 2018
Bread wheat is most cultivated cereal used as food over $95 \%$ of the population. Twenty wheat genotypes were assayed to study the genetic diversity using molecular markers. The seventy-five alleles were identified with a mean of 2.34 alleles per locus using 32 SSR markers. Majority of SSR markers showed a high level of polymorphism. PIC values ranged from 0.05 (WMS-169) to 0.75 (CWM-107), with an average of 0.38 per primer. The RP value of primer ranges from 0.92 (WMC-177) to 1.94 (WMS-169) with an average value of 1.51, which explains the ability of primers to resolve the studied germplasm. According to similarity matrix, genetic similarity value ranged from 0.51 to 0.91 . The lowest genetic similarity was observed between the WH711 and HD2733 genotypes and the maximum similarity was shown by genotype HD2864 with DBW71. Cluster analysis grouped the twenty wheat genotypes into two main clusters with one separate member. Results indicated that wheat cultivars had high genetic diversity that can be used in wheat breeding programs.

\section{Introduction}

Bread wheat (Triticum aestivum L.) belongs to the family Poaceae, is the most commonly cultivated cereal, currently grown in most of parts of world (Abdellatif and Abouzeid, 2011). In term of production it is having second place after rice (Trnka et al., 2014). Along with maize it is major part of food for $95 \%$ population in developing countries. It is used in form of flour provides one fifth of the global required calories and become most preferred over the rice (Wrigley, 2009; Mwale et al., 2016 and Friedrich et al., 2014). In order to feed the world's growing population, the global demand for wheat yields increase by $50 \%$ by 2050 as estimated by Allen et al., (2017). Around the world breeders are working toward the improved grain yield with better quality along with important agronomic traits, therefore the knowledge of the genetic diversity within a germplasm collection has a significant impact for the improvement of crops and useful for production of more efficient crops adapted to diverse conditions (Desheva and Kyosev, 2015). Genetic 
diversity is a kind of fundamental study for crop improvement and plays an important role in generating new plant ideotypes with desired traits, which offers prospects for improving the plant characteristics (Manjarrez-Sandoval et al., 1997; Singh, 1991 and Khan et al., 2015). The estimated genetic diversity has great importance for optimal utilization and conservation of germplasm for plant breeding and other activities (Uddin and Boerner, 2008).

Its assessment helps to tackle the threats of environmental fluctuations and for the effective exploitation of genetic resources in breeding programmes. Wheat is one of the most thoroughly studied crops in terms of genetic polymorphism studies but phylogenetic affinities of Triticum species have not been assessed to date (Khan et al., 2015). So, it is necessary to investigate the genetic diversity in wheat germplasm in order to broaden the genetic variation for future breeding and genetic resource conservation programme.

During the last few decades SSR (microsatellite) markers have been playing an increasing part in genetic studies (Akfirat and Uncuoglu, 2013) permit the fast and high throughput fingerprinting of large numbers of accessions from a germplasm collection in order to assess genetic diversity (Cifci and Yagdi, 2012 and Malik et al., 2013).

They provide new dimension, perfection and accuracy in screening of germplasm (Tar'an et al., 2005). The status of genetic diversity in wheat genotypes assessed by Arora et al., (2014) can be used effectively for future breeding practices. The aim of the present study was done to utilize SSR markers in order to assess the genetic diversity of twenty Indian wheat genotypes. This study was conducted to understand the genetic diversity of Indian wheat genotypes.

\section{Materials and Methods}

This investigation was carried out during the crop seasons 2016 and 2017 at the Field research laboratory and experimental station with proper agronomic practices. The molecular works conducted at Department of Agriculture Biotechnology, Sardar Vallabhbhai Patel University of Agriculture and Technology, Meerut, India $\left(28.99^{\circ} \mathrm{N}\right.$ Latitude and $77.7^{\circ} \mathrm{E}$ Longitude with an altitude of $220 \mathrm{~m}$ above the mean sea level).

\section{Plant material, DNA extraction and SSR analysis}

In present study 20 wheat genotypes (Table 1) were used for the assessment of genetic diversity. Total genomic DNA from fresh leaf of plants was extracted using CTAB method (Murray and Thompson, 1980).

DNA was quantified by spectrophotometer, at 260/280nm absorbance. For PCR reaction, the DNA was diluted in the range between 50$100 \mathrm{ng} / \mu \mathrm{l}$. Total thirty-five SSR markers were used for estimation of genetic diversity were selected randomly from prior reported SSR markers (Gao et al., 2003 and Al-naggara et al., 2013). DNA amplification reaction for SSR was performed in a total volume of $25 \mu \mathrm{l}$.

The components used for reaction mixture are 10x Taq Buffer (2.5 $\mu 1), 10 \mathrm{mM}$ dNTP mix $0.5 \mu 1$, forward primer $0.25 \mu 1$, reverse primer $0.25 \mu 1$, Taq polymerase $0.5 \mu 1$, template $1 \mu 1$ and volume make up to $25 \mu \mathrm{l}$ in $0.2 \mathrm{ml}$ thin walled PCR-tubes with the following thermal program; denatured at $94^{\circ} \mathrm{C}$ for 4 minutes followed by 35 cycles, denature at $94^{\circ} \mathrm{C}$ for 30 second, annealing at $42-58^{\circ} \mathrm{C}$ (depending on TM of SSR primer) for 40 second, extension of primer at $72^{\circ} \mathrm{C}$ for 1 minute followed by final extension at $72^{\circ} \mathrm{C}$ for 10 minutes and hold at $4^{\circ} \mathrm{C}$. The amplified products were separated on $1 \%$ agarose gels and in $1 \mathrm{X}$ TAE 
buffer and DNA fragments were visualized under UV trans illuminator using Alpha Imager gel doc.

\section{Data collection and analysis}

Thirty-five SSR primers were used for generating the reference data for the usefulness of a primer. Zero-one sheet was prepared by scoring SSR primer on the basis of presence (1) and absence (0) in all wheat genotypes for further analysis. Genetic similarities were calculated using the Jaccard similarity coefficient (Jaccard, 1908) metod and dendrogram acquired by clustering according to the Un-weighted Pair Group Method with Arithmetic average (UPGMA) algorism using the NTSYS-pc software version 2.11s (Rohlf, 2000). The resolving power (RP) for each primer was calculated in order to assess the ability of primers to resolve the different varieties by following Prevost and Wilkinson's (1999) method as RP = Ib (band information) and RP was calculated as $1-[2 \times(0.5-p)]$, where $p$ being the proportion of the 20 varieties containing the bands and Polymorphism information content (PIC) values were obtained using the formula developed by Anderson et al., (1993). PIC = 1- $\Sigma \mathrm{Pij} 2$, where Pij is the frequency of $\mathrm{jth}$ allele of ith locus, summed across all the alleles for the locus over all genotypes.

\section{Results and Discussion}

Loss of genetic diversity has become a problem for agriculturally important species. Decrease in genetic variation affects the productivity and adaptability for improvement of bread wheat (Stoeva et al., 2009). Genetic diversity in wheat is becoming narrowed due to modern breeding, which is a problem for adaptation to biotic and abiotic stresses, like salt or drought tolerance (Nasab et al., 2013). The use of molecular markers for the evaluation of genetic diversity is receiving much attention as they allow calculation of genetic distance based on allele frequencies and useful in studying the relationship of closely related lines (Uddin and Boerner, 2008; Huang et al., 2002). Availability of superior and diverse alleles/genes form the basis of genetic improvement of crop plants including wheat (Abouzied et al., 2013) that can help in identification of new cultivars. Haile et al., (2013) reported that SSR markers are more variable than other molecular markers, which are useful tools for the study of genetic diversity of germplasms.

\section{Polymorphism of SSR markers}

Out of 35 tested SSR primers 32 SSR generate clear and reproducible bands have considered for further analysis was polymorphic range from 50.00 to $100 \%$ polymorphism. A total of 75 alleles were amplified by 32 SSR primers in 20 genotypes range from minimum 1 to maximum 5 (Figure 1) with an average of 2.34 alleles per primer. Liu et al., (2005) reported on average 1.9 polymorphic loci per reaction. Wang et al., (2007) analyzed on an average 3.3 alleles per locus by using 26 SSR in 60 durum wheat genotypes.

The assessment of efficiency of molecular markers could be assessed with PIC and RP parameters (Phougat et al., 2017). PIC value was calculated for thirty two polymorphic SSR primers and shown in Table 2 and graphically present in figure (Figure 2).

The PIC value ranges from 0.05 for primer WMS-169 to 0.75 was recorded for the primer CWM-107, with an average of 0.38 for all polymorphic SSR primers. These results are comparable with the results reported by Salem et al., (2015), use 17 polymorphic microsatellite markers to explain the genetic diversity of hexaploid wheat and report PIC value ranged of 0.33 and similar results reported by Sharma et al., (2010) also. 
Table.1 Names of wheat genotypes used in the study

\begin{tabular}{|c|l|l|}
\hline No & Name/ Identity & \multicolumn{1}{|c|}{ Pedigree } \\
\hline $\mathbf{1}$ & DBW17 & CMH 79A.95/3* CNO 79//Raj 3777 \\
\hline $\mathbf{2}$ & DBW71 & Prinia/UP 2425 \\
\hline $\mathbf{3}$ & HD2733 & ATTILA/3/TUI/CARC//CHEN/CHTO/4/ATTILA \\
\hline $\mathbf{4}$ & HD2864 & DL 509-2/ DL 377-8 \\
\hline $\mathbf{5}$ & HD2888 & C 306/T. sphaerococcum//HW 2004 \\
\hline $\mathbf{6}$ & HD3086 & DBW 14/HD 2733//HUW 468 \\
\hline $\mathbf{7}$ & HUW468 & CPAN-1962 / TONI // LIRA'S / PRL'S' \\
\hline $\mathbf{8}$ & K-8027 & HD1969/K852//K852. \\
\hline $\mathbf{9}$ & K-1256 & - \\
\hline 10 & K-9107 & K 8101/K 68 \\
\hline 11 & K-9423 & HP1633/KAL/UP262 \\
\hline 12 & PBW226 & C591/RN//JN/3/CHR/HD1941 \\
\hline 13 & PBW343 & ND/VG 7944//KAL/BB3YACO S/4/VEE\# 5S \\
\hline 14 & PBW396 & CN067/MFD//M0N 'S73/SERI \\
\hline 15 & PBW590 & 594/RAJ3814//W 485 \\
\hline 16 & RAJ3765 & HD 2402/VL 639 \\
\hline 17 & RAJ4246 & - \\
\hline $\mathbf{1 8}$ & UP-2425 & HD2320/UP2263 \\
\hline $\mathbf{1 9}$ & WH711 & ALD 'S'HUAC//HD 2285/3/HFW-17 \\
\hline $\mathbf{2 0}$ & WH1021 & NYOT95/SONAK \\
\hline
\end{tabular}

Table.2 Description of SSR markers employed in the study

\begin{tabular}{|r|c|c|c|c|c|c|c|c|c|c|}
\hline No & Primer name & No. of allele & PIC & RP & No & Primer name & No. of allele & PIC & RP \\
\hline $\mathbf{1}$ & CWM-101 & 1 & 0.51 & 1.40 & $\mathbf{1 7}$ & Xwmc382-2A & 1 & 0.10 & $\mathbf{1 . 9 0}$ \\
\hline $\mathbf{2}$ & CWM-103 & 1 & 0.44 & 1.50 & $\mathbf{1 8}$ & WMS-06 & 3 & 0.25 & $\mathbf{1 . 7 0}$ \\
\hline $\mathbf{3}$ & CWM-105 & 2 & 0.75 & 1.00 & $\mathbf{1 9}$ & WMS-30 & 4 & 0.52 & $\mathbf{1 . 2 5}$ \\
\hline $\mathbf{4}$ & CWM-107 & 2 & 0.64 & 1.06 & $\mathbf{2 0}$ & WMS-108 & 3 & 0.55 & $\mathbf{1 . 1 2}$ \\
\hline $\mathbf{5}$ & CWM-110 & 5 & 0.51 & 1.30 & $\mathbf{2 1}$ & WMS-118 & 2 & 0.40 & $\mathbf{1 . 4 0}$ \\
\hline $\mathbf{6}$ & CWM-112 & 3 & 0.45 & 1.40 & $\mathbf{2 2}$ & WMS-149 & 3 & 0.46 & $\mathbf{1 . 2 0}$ \\
\hline $\mathbf{7}$ & CWM-115 & 2 & 0.19 & 1.80 & $\mathbf{2 3}$ & WMS-169 & 2 & 0.05 & $\mathbf{1 . 9 4}$ \\
\hline $\mathbf{8}$ & CWM-118 & 3 & 0.30 & 1.60 & $\mathbf{2 4}$ & WMC-177 & 3 & 0.74 & $\mathbf{0 . 9 2}$ \\
\hline $\mathbf{9}$ & CWM-119 & 3 & 0.52 & 1.30 & $\mathbf{2 5}$ & WMC-179 & 4 & 0.08 & $\mathbf{1 . 9 2}$ \\
\hline $\mathbf{1 0}$ & CWM-122 & 3 & 0.16 & 1.80 & $\mathbf{2 6}$ & WMS-198 & 1 & 0.19 & $\mathbf{1 . 8 0}$ \\
\hline $\mathbf{1 1}$ & XGWM-11-1D & 1 & 0.10 & 1.90 & $\mathbf{2 7}$ & WMC-235 & 2 & 0.10 & $\mathbf{1 . 9 0}$ \\
\hline $\mathbf{1 2}$ & XGWM-260-7A & 4 & 0.25 & 1.60 & $\mathbf{2 8}$ & WMS-304 & 1 & 0.10 & $\mathbf{1 . 9 0}$ \\
\hline $\mathbf{1 3}$ & XGWM276-7A & 1 & 0.44 & 1.50 & $\mathbf{2 9}$ & WMC-307 & 1 & 0.27 & $\mathbf{1 . 7 0}$ \\
\hline $\mathbf{1 4}$ & XGWM-350-7A & 3 & 0.50 & 1.30 & $\mathbf{3 0}$ & WMC-322 & 2 & 0.47 & $\mathbf{1 . 2 5}$ \\
\hline $\mathbf{1 5}$ & XGWM-573-7B & 2 & 0.60 & 1.20 & $\mathbf{3 1}$ & WMS-375 & 3 & 0.44 & $\mathbf{1 . 4 6}$ \\
\hline $\mathbf{1 6}$ & XGWM-635-7D & $\mathbf{2}$ & $\mathbf{0 . 1 8}$ & $\mathbf{1 . 8 0}$ & $\mathbf{3 2}$ & WMC-445 & $\mathbf{2}$ & $\mathbf{0 . 4 5}$ & $\mathbf{1 . 4 5}$ \\
\hline
\end{tabular}


Fig.1 SSR primer CWM-103(a) and CWM-107(b), profiling pattern of 20 wheat varieties along with $1 \mathrm{~Kb}(\mathrm{M} 1)$ and $100 \mathrm{bp}(\mathrm{M} 2)$ DNA ladder
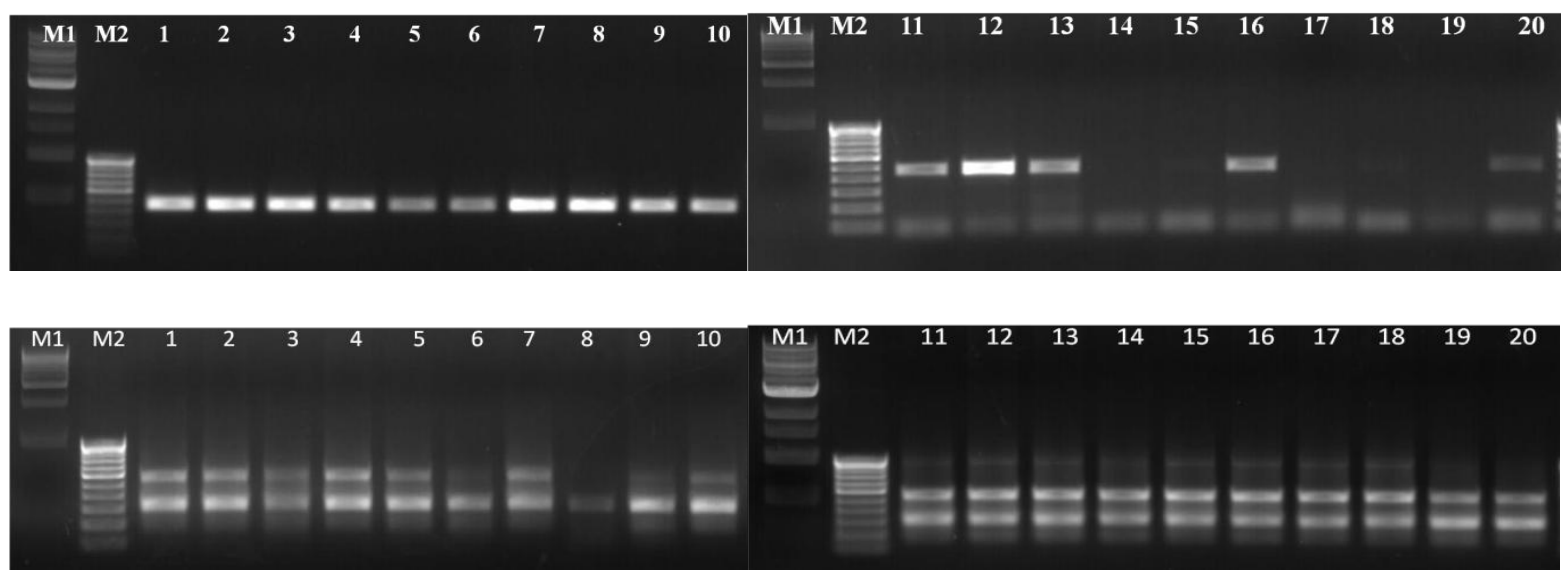

Fig.2 Graphical representation of PIC value of SSR markers

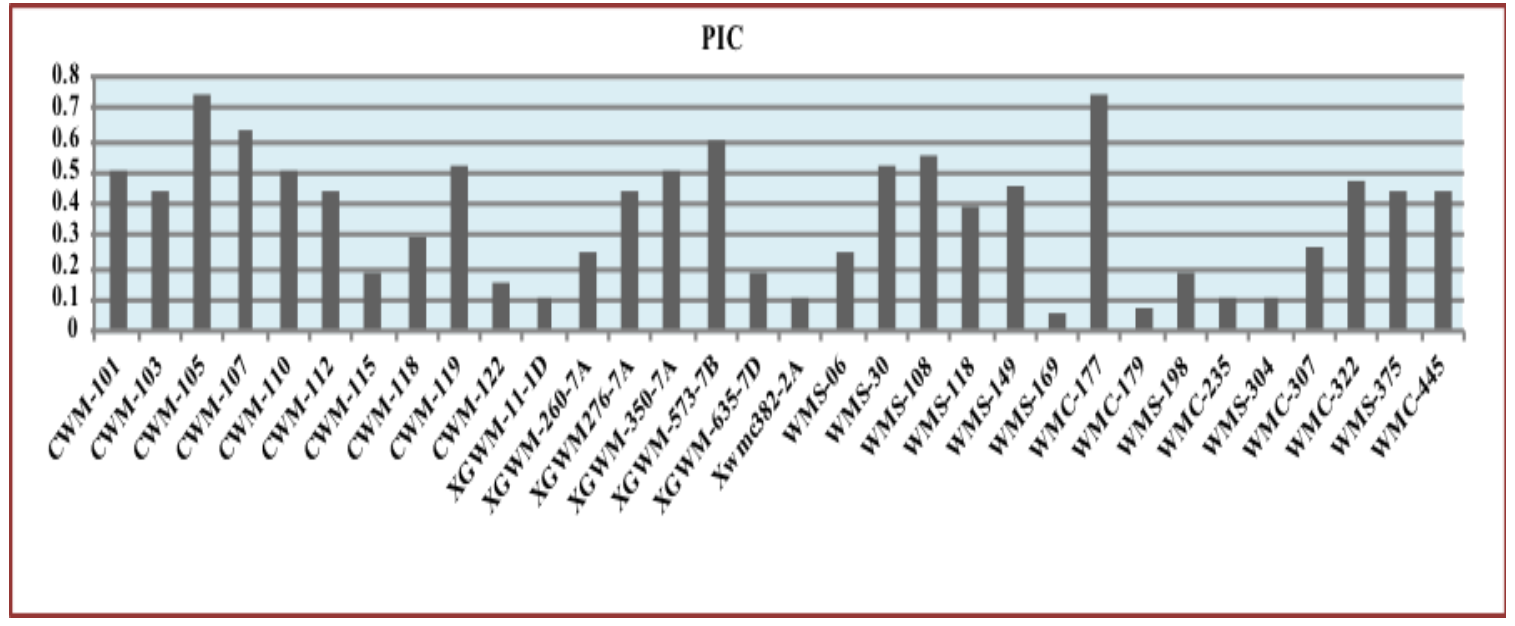

Fig.3 Graphical representation of resolving power (RP) value of SSR markers

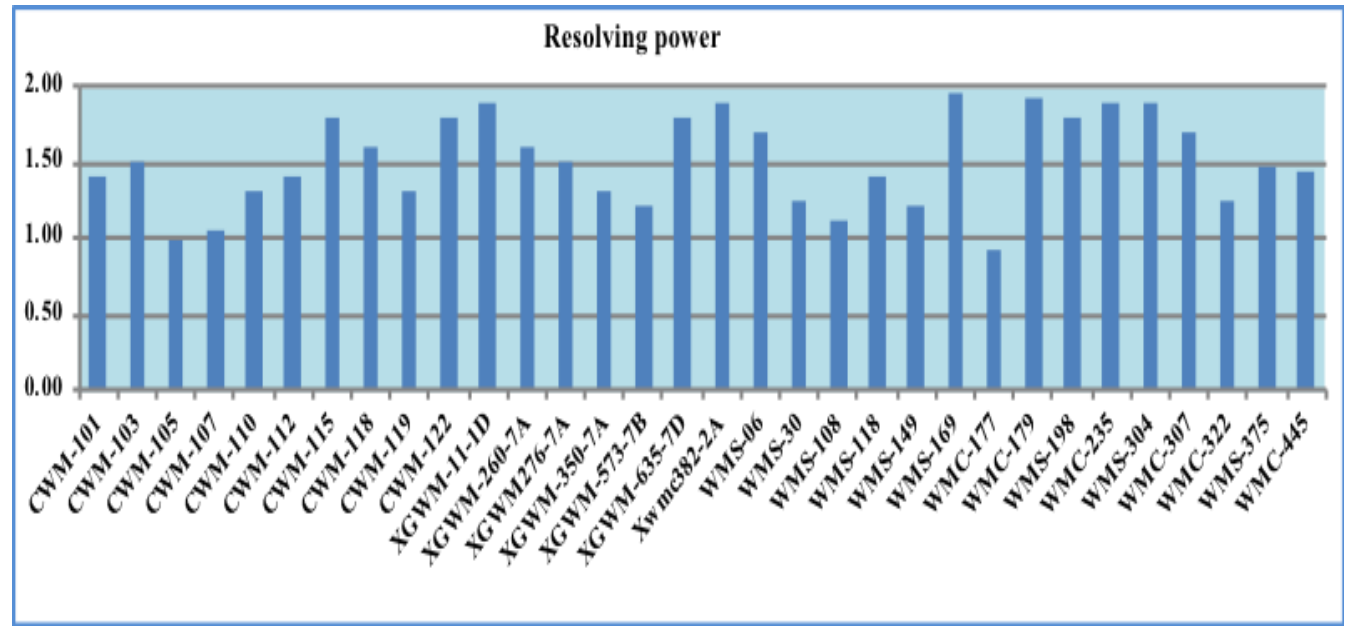


Fig.4 Dendrogram from UPGMA analysis based on Jaccard similarity coefficient of 20 Indian wheat cultivars



Tekeu et al., (2017) aimed to estimate the levels and genetic structure within 17 bread wheat variety using 11 microsatellite markers revealing 77 alleles. PIC value between 0.16 and 0.91 for SSRs was also reported by Bohn et al., (1999). Ahmad, (2002) evaluated 13 wheat cultivars of diverse origin using 43 SSR markers and report similar results also. Marmar et al., (2013) carried out research to screen 12 wheat cultivars to study genetic diversity with 24 allele specific SSR markers showing polymorphism information content ranging from 0.16 to 0.89 . In the present study besides primer CWM-105, the other primer like CWM-107, WMC-177 and XGWM-573-7B also show higher PIC value. The higher mean PIC value indicated the informativeness of the primers pairs in detecting genetic diversity and can be used in future studies in the field of taxonomical and genetic resource management. The resolving power (RP) of primer explains the ability of primers to resolve the studied germplasms and ability of a primer to distinguish between large numbers of genotypes (Provost and
Wilkinson, 1999; Ablett et al., 2006). The resolving power of 32 polymorphic SSR primers varies from 0.92 (WMC-177) to 1.94 (WMS-169) with an average value of 1.51 (Table 2) and graphically present in figure 3 ). Singh et al., (2017) reports resolving power with an average value of 1.79 in wheat genotypes.

\section{Cluster analysis}

The cluster analysis based on the UPGMA method, SSR primers allowed the discrimination of cultivars and represent the estimated relations between different genotypes (Singh et al., 2017). To present the genetic relationship a dendrogram was constructed, which generate two major groups i.e. cluster I and cluster II groups (Figure 4). The genotype WH711 did not grouped in any cluster and stays separated at the one end of the cluster. The group I subdivided into two sub clusters viz. cluster Ia and cluster Ib. The sub cluster Ia further divided into small clusters includes 7 genotypes namely 
DBW17, DBW 71, HD2864, HD2733, HUW468, K8027, HD3086 and cluster Ib includes 3 genotypes HD2888, K9107 and K1256. The cluster II subdivided into two sub cluster (IIa and IIb). The sub cluster IIa includes 3 genotypes namely K9423, WH1021, PBW343 and subcluster IIb includes PBW396, UP2425, RAJ3765, PBW590, PBW226 and RAJ4246 which are further grouped into small clusters. Grouping of wheat genotypes into different clusters have relevance to the future breeding programs (Sharma et al., 2010). Genetic similarity value for all the 20 genotypes ranged from 0.51 to 0.91 . The minimum similarity exhibited by genotype WH711 with HD2733 and the maximum similarity was shown by genotype HD2864 with DBW71. The distribution of similarity coefficient is shown in figure 4. Higher similarity values provide greater confidence for the assessment of genetic diversity and relationships in related genotypes. Islam et al., (2012) report microsatellite markers are helpful to characterize and discriminate the diversity within the wheat genotypes. For evaluating genetic relationships diversity analysis is a key factor, which is use in breeding of improved varieties (Al-Doss et al., 2011).

In this investigation, SSR markers showed a high level of polymorphism and are more informative in hexaploid wheat. The genetic diversity levels observed in bread wheat that cultivated in India would be useful indicators if such an approach is planned for the wheat genome and outcome of this research could provide appropriate guidelines for plant breeders towards the implementation of future crop improvement programs for proper management of the wheat cultivars.

\section{References}

Abdellatif, K. F., and Abou Zeid, H. M. (2011). Assessment of genetic diversity of Mediterranean bread wheat using Randomly Amplified Polymorphic DNA (RAPD) markers. Journal of Genetic Engineering and Biotechnology, 9(2): 157-163.

Ablett, G., Hill, H. and Henry, R. J. (2006). Sequence polymorphism discovery in wheat microsatellite flanking regions using pyrophosphate sequencing. Molecular Breeding, 17(3): 281-289.

Abouzied, H. M., Eldemery, S. M. and Abdellatif, K. F. (2013). SSR-Based Genetic Diversity Assessment in Tetraploid and Hexaploid Wheat Populations. British Biotechnology Journal, 3: 390- 404.

Ahmad, M. (2002). Assessment of genomic diversity among wheat genotypes as determined by simple sequence repeats. Genome, 45(4): 646-651.

Akfirat, F. S. and Uncuoglu, A. A. (2013). Genetic diversity of winter wheat (Triticum aestivum L.) revealed by SSR markers. Biochemical genetics, 51(3-4): 223-229.

Al-Doss, A. A., Elshafei, A. A., Moustafa, K. A., Saleh, M. and Barakat, M. N. (2011). Comparative analysis of diversity based on morphoagronomic traits and molecular markers in durum wheat under heat stress. African Journal of Biotechnology, 10(19): 3671-3681.

Allen, A. M., Winfield, M. O., Burridge, A. J., Downie, R. C., Benbow, H. R., Barker, G. L., Wilkinson, P.A., Coghill, J., Waterfall, C., Davassi, A. and Scopes, G. (2017). Characterization of a Wheat Breeders' Array suitable for high- throughput SNP genotyping of global accessions of hexaploid bread wheat (Triticum aestivum). Plant biotechnology journal, 15(3): 390-401.

Al-Naggara. M.M., Sobieh S.E.S., Attam. M.M. and Al-Azab K.F. (2013). Unique ssr markers for drought tolerance in newly-developed bread wheat mutants. 
World Research Journal of Agronomy, 2(1): 015-025.

Anderson, J. A., Churchill, G. A., Autrique, J. E., Tanksley, S. D. and Sorrells, M. E. (1993). Optimizing parental selection for genetic linkage maps. Genome, 36(1): 181-186.

Arora, A., Kundu, S., Dilbaghi, N., Sharma, I. and Tiwari, R. (2014). Population structure and genetic diversity among Indian wheat varieties using microsatellite (SSR) markers. Australian Journal of Crop Science, 8(9): 1281.

Bohn, M., Utz, H. F. and Melchinger, A. E. (1999). Genetic similarities among winter wheat cultivars determined on the basis of RFLPs, AFLPs, and SSRs and their use for predicting progeny variance. Crop science, 39(1): 228-237.

Cifci, E.A. and Yagdi, K. (2012). Study of genetic diversity in wheat (Triticum aestivum) varieties using random amplified polymorphic DNA (RAPD) analysis. Turkish Journal of Field Crops, 17: 91-95.

Desheva, G. and Kyosev, B. (2015). Genetic diversity assessment of common winter wheat (Triticum aestivum L.) genotypes. Emirates Journal of Food and Agriculture, 27(3): 283.

Friedrich, C., Longin, H. and Reif, C.J. (2014). Redesigning the exploitation of wheat genetic resources. Trends in plant science, 19(10): 631-636.

Gao, L., Tang, J., Li, H., and Jia, J. (2003). Analysis of microsatellites in major crops assessed by computational and experimental approaches. Molecular Breeding, 12(3): 245-261.

Haile, J. K., Hammer, K., Badebo, A., Nachit, M. M., and Röder, M. S. (2013). Genetic diversity assessment of Ethiopian tetraploid wheat landraces and improved durum wheat varieties using microsatellites and markers linked with stem rust resistance. Genetic resources and crop evolution, 60(2): 513-527.

Huang, X., Börner, A., Röder, M. and Ganal, M. (2002). Assessing genetic diversity of wheat (Triticum aestivum L.) germplasm using microsatellite markers. Theoretical and Applied Genetics, 105(5): 699-707.

Islam, S., Haque, M. S., Emon, R. M., Islam, M. M. and Begum, S. N. (2012). Molecular characterization of wheat (Triticum aestivum L.) genotypes through SSR markers. Bangladesh Journal of Agricultural Research, 37(3): 389-398.

Jaccard, P. (1908). Nouvelles researches sur la distribution florale. Bulletin Society Vaud Science National, 44: 223-270.

Khan, M. K., Pandey, A., Thomas, G., Akkaya, M. S., Kayis, S. A., Ozsensoy, Y., Hamurcu, M., Gezgin, S., Topal, A. and Hakki, E. E. (2015). Genetic diversity and population structure of wheat in India and Turkey. AoB Plants, 7, plv083.

Liu, Z. H., Anderson, J. A., Hu, J., Friesen, T. L., Rasmussen, J. B. and Faris, J. D. (2005). A wheat intervarietal genetic linkage map based on microsatellite and target region amplified polymorphism markers and its utility for detecting quantitative trait loci. Theoretical and Applied Genetics, 111(4): 782-794.

Malik, R., Tiwari, R., Arora, A., Kumar, P., Sheoran, S., Sharma, P., Singh, R., Tiwari, V. and Sharma, I. (2013). Genotypic characterization of elite Indian wheat genotypes using molecular markers and their pedigree analysis. Australian Journal of Crop Science, 7(5): 561.

Manjarrez-Sandoval, P., Carter, T. E., Webb, D. M. and Burton, J. W. (1997). RFLP genetic similarity estimates and coefficient of parentage as genetic 
variance predictors for soybean yield. Crop science, 37(3): 698-703.

Marmar, A., Baenziger, S., Dweikat, I. and El Hussein, A. A. (2013). Preliminary screening for water stress tolerance and genetic diversity in wheat (Triticum aestivum L.) cultivars from Sudan. Journal of Genetic Engineering and Biotechnology, 11(2): 87-94.

Murray, M. G. and Thompson, W. F. (1980). Rapid isolation of high molecular weight plant DNA. Nucleic acids research, 8(19): 4321-4326.

Mwale, V. M., Tang, X. and Chilembwe, E. (2016). Assessment of genetic diversity among sixty bread wheat (Triticum aestivum) cultivars using microsatellite markers. African Journal of Biotechnology, 15(21): 960-973.

Nasab, S., Mohammadi Nejad, G. and Nakhoda, B. (2013). Assessing genetic diversity of promising wheat (Triticum aestivum L.) lines using microsatellite markers linked with salinity tolerance. Journal of Plant Molecular Breeding, 1(2): 28-39.

Phougat, D., Singh, V., Panwar, I. S. and Yashveer, S. (2017). Evaluating Microsatellite Markers for the use in Genetic Analysis of Hexaploid Wheat Varieties and Elite Lines. Vegetos-An International Journal of Plant Research, 30(special): 132-138.

Prevost, A. and Wilkinson, M. J. (1999). A new system of comparing PCR primers applied to ISSR fingerprinting of potato cultivars. Theoretical and applied Genetics, 98(1): 107-112.

Rohlf, F. J. (2000). NTSYS-PC numerical taxonomy and multivariate system, version 2.1 Applied Biostatistics Inc. New York.

Salem, K. F., Röder, M. S. and Börner, A. (2015). Assessing genetic diversity of Egyptian hexaploid wheat (Triticum aestivum L.) using microsatellite markers. Genetic resources and crop evolution, 62(3): 377-385.

Sharma, J., Goyal, N., Singh, A., Pallavi, J. K., Sonah, H., and Gupta, P. (2010). Assessment of Genetic Relationships among Bread Wheat (Triticum aestivum L. em. Thell.) Genotypes using Microsatellite Markers. International Journal of Applied, 5(5): 575-582.

Singh, B., Goswami, A. and Vaishali. (2017). Study of Genetic Diversity in Okra [Abelmoschus esculentus (L.) Moench]. Vegetos-An International Journal of Plant Research, 30(special): 109-115.

Singh, N. P., Vaishali., Goswami, A., and Singh, B. (2017). Assessing Genetic Diversity of Wheat (Triticum aestivum L.) using Simple Sequence Repeats Markers. Vegetos-An International Journal of Plant Research, 30(special), 169-175.

Singh, S. P. (1991). Breeding for seed yield. Common Beans: Research for Crop Improvement, 383-443.

Stoeva, I., Chamurliysky, P. and Tsenov, N. (2009). Investigation on Bulgarian and foreign common winter wheat varieties and lines with a view of their using in breeding for productivity and quality. Field Crops Studies, 5(2): 253-260.

Tar'an, B., Zhang, C., Warkentin, T., Tullu, A. and Vandenberg, A. (2005). Genetic diversity among varieties and wild species accessions of pea (Pisum sativum L.) based on molecular markers, and morphological and physiological characters. Genome, 48(2), 257-272.

Tekeu, H., Ngonkeu, E. M., Djocgoue, F. P., Ellis, A., Lendzemo, V., Springfield, L., Moulin, L., Klonowska, A., Diouf, D., Botes, W.C. and Bena, G. (2017). Genetic diversity of Cameroonian bread wheat (Triticum aestivum L.) cultivars revealed by microsatellite markers. 
African Journal of Biotechnology, 16(36), 1832-1839.

Trnka, M., Rötter, R. P., Ruiz-Ramos, M., Kersebaum, K. C., Olesen, J. E., Žalud, Z. and Semenov, M. A. (2014). Adverse weather conditions for European wheat production will become more frequent with climate change. Nature Climate Change, 4(7): 637.

Uddin, M. S. and Boerner, A. (2008). Genetic diversity in hexaploid and tetraploid wheat genotypes using microsatellite markers. Plant Tissue Culture and Biotechnology, 18(1), 65-73.

Wang, H. Y., Wei, Y. M., Yan, Z. H. and Zheng, Y. L. (2007). EST-SSR DNA polymorphism in durum wheat (Triticum durum L.) collections. Journal of Applied Genetics, 48(1), 3542.

Wrigley, C. W. (2009). Wheat: a unique grain for the world. Wheat: chemistry and technology, (Ed. 4): 1-17.

Yagdi, E. A. C. K. (2012). Study of genetic diversity in wheat (Triticum aestivum) varities using Random Amplified Polymorphic DNA (RAPD) analysis. Turkish Journal of Field Crops, 17(1), 91-95.

\section{How to cite this article:}

Vandana Sharma, Vaishali, Pushpendra Kumar, Manoj Kumar Yadav and Pooran Chand. 2018. Assessment of Genetic Diversity among Twenty Indian Wheat (Triticum aestivum L.) Cultivars using Simple Sequence Repeat (SSR) Markers. Int.J.Curr.Microbiol.App.Sci. 7(03): 17081717. doi: https://doi.org/10.20546/ijcmas.2018.703.202 